International Journal of Pure and Applied Mathematics

Volume 93 No. 1 2014, 121-134

ISSN: 1311-8080 (printed version); ISSN: 1314-3395 (on-line version)

url: http://www.ijpam.eu

doi: http://dx.doi.org/10.12732/ijpam.v93i1.10

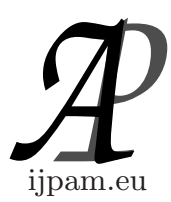

\title{
JOIN-MEET AND MEET-JOIN PRESERVING MAPS
}

\author{
Yong Chan Kim \\ Department of Mathematics \\ Gangneung-Wonju University \\ Gangneung, Gangwondo 210-702, KOREA
}

\begin{abstract}
We investigate the properties of join-meet and meet-join preserving maps in complete residuated lattice. In particular, we give their examples.

AMS Subject Classification: 03E72, 03G10, 06A15, 06F07

Key Words: join-meet and meet-join preserving maps, fuzzy preorder, complete residuated lattices
\end{abstract}

\section{Introduction}

Pawlak $[6,7]$ introduced the rough set theory as a formal tool to deal with imprecision and uncertainty in the data analysis. Hájek [2] introduced a complete residuated lattice which is an algebraic structure for many valued logic. By using the concepts of lower and upper approximation operators, information systems and decision rules are investigated in complete residuated lattices $[1,2,8,9]$. Bělohlávek [1] developed the notion of fuzzy contexts using Galois connections with $R \in L^{X \times Y}$ on a complete residuated lattice. Zhang [10,11] introduced the fuzzy complete lattice which is defined by join and meet on fuzzy posets. It is an important mathematical tool for algebraic structure of fuzzy contexts [1,4-6]. Kim [3] show that join (resp. meet, meet join, join meet) preserving maps and upper (resp. lower, meet join, join meet) approximation maps are equivalent in complete residuated lattices.

In this paper, we investigate the properties of join-meet and meet-join preserving maps in complete residuated lattice. In particular, we give their examples.

Received: January 13, 2014

(C) 2014 Academic Publications, Ltd. url: www.acadpubl.eu 


\section{Preliminaries}

Definition 1. [1,2] A structure $(L, \vee, \wedge, \odot, \rightarrow, \perp, \top)$ is called a complete residuated lattice iff it satisfies the following properties:

(L1) $(L, \vee, \wedge, \perp, \top)$ is a complete lattice where $\perp$ is the bottom element and $\top$ is the top element;

(L2) $(L, \odot, \top)$ is a monoid;

(L3) adjointness properties,i.e.

$$
x \leq y \rightarrow z \text { iff } x \odot y \leq z .
$$

A map ${ }^{*}: L \rightarrow L$ defined by $a^{*}=a \rightarrow \perp$ is called strong negations if $a^{* *}=a$.

$$
\top_{x}(y)=\left\{\begin{array}{ll}
\top, & \text { if } y=x, \\
\perp, & \text { otherwise. }
\end{array} \top_{x}^{*}(y)= \begin{cases}\perp, & \text { if } y=x, \\
\top, & \text { otherwise. }\end{cases}\right.
$$

In this paper, we assume that $\left(L, \vee, \wedge, \odot, \rightarrow,{ }^{*}, \perp, \top\right)$ be a complete residuated lattice with a strong negation *

Definition 2. $[10,11]$ Let $X$ be a set. A function $e_{X}: X \times X \rightarrow L$ is called:

(E1) reflexive if $e_{X}(x, x)=1$ for all $x \in X$,

(E2) transitive if $e_{X}(x, y) \odot e_{X}(y, z) \leq e_{X}(x, z)$, for all $x, y, z \in X$,

(E3) if $e_{X}(x, y)=e_{X}(y, x)=1$, then $x=y$.

If $e$ satisfies (E1) and (E2), $\left(X, e_{X}\right)$ is a fuzzy preorder set. If $e$ satisfies (E1), (E2) and (E3), $\left(X, e_{X}\right)$ is a fuzzy partially order set (simply, fuzzy poset).

Example 3. (1) We define a function $e_{L}: L \times L \rightarrow L$ as $e_{L}(x, y)=x \rightarrow y$. Then $\left(L, e_{L}\right)$ is a fuzzy poset.

(2) We define a function $e_{L^{X}}: L^{X} \times L^{X} \rightarrow L$ as $e_{L^{X}}(A, B)=\bigwedge_{x \in X}(A(x) \rightarrow$ $B(x))$. Then $\left(L^{X}, e_{L^{X}}\right)$ is a fuzzy poset from Lemma $10(9)$.

Definition 4. $[10,11]$ Let $\left(X, e_{X}\right)$ be a fuzzy poset and $A \in L^{X}$.

(1) A point $x_{0}$ is called a join of $A$, denoted by $x_{0}=\sqcup A$, if it satisfies

(J1) $A(x) \leq e_{X}\left(x, x_{0}\right)$,

$(\mathrm{J} 2) \bigwedge_{x \in X}\left(A(x) \rightarrow e_{X}(x, y)\right) \leq e_{X}\left(x_{0}, y\right)$.

A point $x_{1}$ is called a meet of $A$, denoted by $x_{1}=\sqcap A$, if it satisfies

(M1) $A(x) \leq e_{X}\left(x_{1}, x\right)$,

(M2) $\bigwedge_{x \in X}\left(A(x) \rightarrow e_{X}(y, x)\right) \leq e_{X}\left(y, x_{1}\right)$.

Remark 5. Let $\left(X, e_{X}\right)$ be a fuzzy poset and $A \in L^{X}$. 
(1) If $x_{0}$ is a join of $A$, then it is unique because $e_{X}\left(x_{0}, y\right)=e_{X}\left(y_{0}, y\right)$ for all $y \in X$, put $y=x_{0}$ or $y=y_{0}$, then $e_{X}\left(x_{0}, y_{0}\right)=e_{X}\left(y_{0}, x_{0}\right)=\top$ implies $x_{0}=y_{0}$. Similarly, if a meet of $A$ exist, then it is unique.

(2) $x_{0}$ is a join of $A$ iff $\bigwedge_{x \in X}\left(A(x) \rightarrow e_{X}(x, y)\right)=e_{X}\left(x_{0}, y\right)$.

(3) $x_{1}$ is a meet of $A$ iff $\bigwedge_{x \in X}\left(A(x) \rightarrow e_{X}(y, x)\right)=e_{X}\left(y, x_{1}\right)$.

Remark 6. Let $\left(L, e_{L}\right)$ be a fuzzy poset and $A \in L^{L}$.

(1) Since $x_{0}$ is a join of $A$ iff $\bigwedge_{x \in L}\left(A(x) \rightarrow e_{L}(x, y)\right)=\bigwedge_{x \in L}(A(x) \rightarrow$ $(x \Rightarrow y))=\bigvee_{x \in L}(x \odot A(x)) \rightarrow y=e_{L}\left(x_{0}, y\right)=x_{0} \rightarrow y$, then $x_{0}=\sqcup A=$ $\bigvee_{x \in L}(x \odot A(x))$.

(2) Since $x_{0}$ is a join of $A$ iff $\bigwedge_{x \in L}\left(A(x) \rightarrow e_{L}(x, y)=\bigwedge_{x \in L}(A(x) \rightarrow(y \rightarrow\right.$ $x))=\bigwedge_{x \in L}(y \rightarrow(A(x) \rightarrow x))=y \rightarrow \bigwedge_{x \in L}(A(x) \rightarrow x)=y \rightarrow \sqcap A$, then $\sqcap A=\bigwedge_{x \in L}(A(x) \rightarrow x)$.

Remark 7. Let $\left(L^{X}, e_{L^{X}}\right)$ be a fuzzy poset and $\Phi \in L^{L^{X}}$.

(1) Since $\bigwedge_{A \in L^{X}}\left(\Phi(A) \rightarrow e_{L^{X}}(A, B)\right)=e_{L^{X}}\left(\bigvee_{A \in L^{X}}(\Phi(A) \odot A), B\right)=$ $e_{L^{X}}(\sqcup \Phi, B)$, then $\sqcup \Phi=\bigvee_{A \in L^{X}}(\Phi(A) \odot A)$.

(2) Since $\bigwedge_{A \in L^{X}}\left(\Phi(A) \rightarrow e_{L^{X}}(B, A)=\bigwedge_{A \in L^{X}} e_{L^{X}}(B,(\Phi(A) \rightarrow A))=\right.$ $e_{L^{X}}\left(B, \bigwedge_{A \in L^{X}}(\Phi(A) \rightarrow A)\right)$, then $\sqcap \Phi=\bigwedge_{A \in L^{X}}(\Phi(A) \rightarrow A)$.

Definition 8. $[10,11]$ Let $\left(L^{X}, e_{L^{X}}\right)$ and $\left(L^{Y}, e_{L^{Y}}\right)$ be fuzzy posets.

(1) $\mathcal{K}: L^{X} \rightarrow L^{Y}$ is a join-meet preserving map if $\mathcal{K}(\sqcup \Phi)=\sqcap \mathcal{K} \rightarrow(\Phi)$ for all $\Phi \in L^{L^{X}}$, where $\mathcal{K}^{\rightarrow}(\Phi)(B)=\bigvee_{\mathcal{K}(A)=B} \Phi(A)$.

(2) $\mathcal{M}: L^{X} \rightarrow L^{Y}$ is a meet-join preserving map if $\mathcal{M}(\sqcap \Phi)=\sqcup \mathcal{M}^{\rightarrow}(\Phi)$ for all $\Phi \in L^{L^{X}}$, where $\mathcal{M}^{\rightarrow}(\Phi)(B)=\bigvee_{\mathcal{M}(A)=B} \Phi(A)$.

Theorem 9. [3] Let $X$ and $Y$ be two sets. Let $\left(L^{X}, e_{L^{X}}\right)$ and $\left(L^{Y}, e_{L^{Y}}\right)$ be fuzzy posets. Then the following statements are equivalent:

(1) $\mathcal{K}: L^{X} \rightarrow L^{Y}$ is a join-meet preserving map iff $\mathcal{K}(\alpha \odot A)=\alpha \rightarrow \mathcal{K}(A)$ and $\mathcal{K}\left(\bigvee_{i \in I} A_{i}\right)=\bigwedge_{i \in I} \mathcal{K}\left(A_{i}\right)$ for all $A, A_{i} \in L^{X}$, and $\alpha \in L$.

(2) $\mathcal{M}: L^{X} \rightarrow L^{Y}$ is a meet-join preserving map iff $\mathcal{M}(\alpha \rightarrow A)=\alpha \rightarrow$ $\mathcal{J}(A)$ and $\mathcal{M}\left(\bigwedge_{i \in I} A_{i}\right)=\bigvee_{i \in I} \mathcal{M}\left(A_{i}\right)$ for all $A, A_{i} \in L^{X}$, and $\alpha \in L$.

Lemma 10. [1,2] Let $\left(L, \vee, \wedge, \odot, \rightarrow,^{*}, \perp, \top\right)$ be a complete residuated lattice with a strong negation ${ }^{*}$. For each $x, y, z, x_{i}, y_{i} \in L$, the following properties hold.

$(1) \odot$ is isotone in both arguments.

$(2) \rightarrow$ is antitone in the first and isotone in the second argument.

(3) $x \rightarrow y=\top$ iff $x \leq y$.

(4) $x \rightarrow \top=\top$ and $\top \rightarrow x=x$.

(5) $x \odot y \leq x \wedge y$.

(6) $x \odot\left(\bigvee_{i \in \Gamma} y_{i}\right)=\bigvee_{i \in \Gamma}\left(x \odot y_{i}\right)$ and $\left(\bigvee_{i \in \Gamma} x_{i}\right) \odot y=\bigvee_{i \in \Gamma}\left(x_{i} \odot y\right)$. 
(7) $x \rightarrow\left(\bigwedge_{i \in \Gamma} y_{i}\right)=\bigwedge_{i \in \Gamma}\left(x \rightarrow y_{i}\right)$ and $\left(\bigvee_{i \in \Gamma} x_{i}\right) \rightarrow y=\bigwedge_{i \in \Gamma}\left(x_{i} \rightarrow y\right)$.

(8) $\left.\bigvee_{i \in \Gamma} x_{i} \rightarrow \bigvee_{i \in \Gamma} y_{i}\right) \geq \bigwedge_{i \in \Gamma}\left(x_{i} \rightarrow y_{i}\right)$.

(9) $(x \rightarrow y) \odot x \leq y$ and $(y \rightarrow z) \odot(x \rightarrow y) \leq(x \rightarrow z)$.

(10) $x \rightarrow y \leq(y \rightarrow z) \rightarrow(x \rightarrow z)$.

(11) $\bigwedge_{i \in \Gamma} x_{i}^{*}=\left(\bigvee_{i \in \Gamma} x_{i}\right)^{*}$ and $\bigvee_{i \in \Gamma} x_{i}^{*}=\left(\bigwedge_{i \in \Gamma} x_{i}\right)^{*}$

(12) $(x \odot y) \rightarrow z=x \rightarrow(y \rightarrow z)=y \rightarrow(x \rightarrow z)$ and $(x \odot y)^{*}=x \rightarrow y^{*}$.

(13) $x^{*} \rightarrow y^{*}=y \rightarrow x$ and $(x \rightarrow y)^{*}=x \odot y^{*}$.

\section{Join-Meet and Meet-Join Preserving Maps}

Theorem 11. Let $\left(L^{X}, e_{L^{X}}\right)$ be a fuzzy poset. Let $\mathcal{K}, \mathcal{K}^{-1}: L^{X} \rightarrow L^{X}$ be join-meet preserving maps such that $\mathcal{K}^{-1}\left(\top_{x}\right)(y)=\mathcal{K}\left(\top_{y}\right)(x)$ for each $x, y \in X$. Let $\mathcal{M}, \mathcal{M}^{-1}: L^{X} \rightarrow L^{X}$ be meet preserving maps such that $\mathcal{M}^{-1}\left(\top_{x}^{*}\right)(y)=$ $\mathcal{M}\left(\top_{y}^{*}\right)(x)$ and $\mathcal{K}^{*}\left(\top_{x}\right)(y)=\mathcal{M}\left(\top_{x}^{*}\right)(y)$ for each $x, y \in X$. For $x, y \in X, \alpha \in L$ and $A, B \in L^{X}$, we have the following properties.

(1) $\mathcal{K}(A)(y)=\bigwedge_{x}\left(A(x) \rightarrow \mathcal{K}\left(\top_{x}\right)(y)\right.$ and

$$
\mathcal{K}^{-1}(A)(y)=\bigwedge_{x}\left(A(x) \rightarrow \mathcal{K}^{-1}\left(\top_{x}\right)(y)=\bigwedge_{x}\left(A(x) \rightarrow \mathcal{K}\left(\top_{y}\right)(x)\right)\right.
$$

(2) $\mathcal{M}(A)(y)=\bigvee_{x}\left(A^{*}(x) \odot \mathcal{M}\left(\top_{x}^{*}\right)(y)\right)$ and $\mathcal{M}^{-1}(A)(y)=\bigvee_{x}\left(A^{*}(x) \odot\right.$ $\left.\mathcal{M}^{-1}\left(\top_{x}^{*}\right)(y)\right)$.

(3) $\mathcal{M}(\top)=\mathcal{M}^{-1}(\top)=\perp$ and $\mathcal{M}(\perp)=\mathcal{K}^{-1}(\perp)=\top$.

(4) $\mathcal{M}(A)=\left(\mathcal{K}\left(A^{*}\right)\right)^{*}$ and $\mathcal{M}(A)=\left(\mathcal{M}\left(A^{*}\right)\right)^{*}$.

(5) $\mathcal{K}(\alpha \rightarrow A) \geq \alpha \odot \mathcal{K}(A)$ and $\mathcal{M}(\alpha \odot A) \leq \alpha \rightarrow \mathcal{M}(A)$.

(6) $\mathcal{M}\left(\top_{x} \rightarrow \alpha\right)(y)=\mathcal{M}\left(\top_{x}^{*}\right)(y) \odot \alpha^{*}=\mathcal{K}^{*}\left(\top_{x} \odot \alpha^{*}\right)(y)$.

(7) $\bigwedge_{\alpha \in L}((A(y) \rightarrow \alpha) \rightarrow \alpha)=A(y)$.

(8) $\mathcal{K}\left(A^{*}\right)=\bigwedge_{\alpha \in L}\left(\mathcal{M}\left(A \odot \alpha^{*}\right) \rightarrow \alpha\right)$.

(9) $\mathcal{K}(\alpha \odot A)=\alpha \rightarrow \mathcal{K}(A)=\mathcal{M}\left(A^{*}\right) \rightarrow \alpha^{*}$.

(10) $\mathcal{M}(\alpha \odot A) \leq \mathcal{K}\left(A^{*}\right) \rightarrow \alpha^{*}$.

(11) $e_{L^{X}}(B, \mathcal{K}(A))=e_{L^{X}}\left(A, \mathcal{K}^{-1}(B)\right)$ and

$$
e_{L^{X}}(\mathcal{M}(A), B)=e_{L^{X}}\left(\mathcal{M}^{-1}(B), A\right) .
$$

(12) $e_{L^{X}}(B, \mathcal{K}(A))=e_{L^{X}}\left(\mathcal{M}^{-1}\left(B^{*}\right), A^{*}\right)$ and

$$
e_{L^{X}}(\mathcal{M}(A), B)=e_{L^{X}}\left(A^{*}, \mathcal{K}^{-1}\left(B^{*}\right)\right) .
$$

(13) $e_{L^{X}}(A, B) \leq e_{L^{X}}(\mathcal{K}(B), \mathcal{K}(A))$.

(14) $e_{L^{X}}(A, B) \leq e_{L^{X}}(\mathcal{M}(B), \mathcal{M}(A))$. 
Proof. (1) For $A=\bigvee_{x \in X}\left(A(x) \odot \top_{x}\right)$, by Theorem 9(1), we have

$$
\begin{aligned}
\mathcal{K}(A)(y) & =\mathcal{K}\left(\bigvee_{x \in X}\left(A(x) \odot \top_{x}\right)\right)(y)=\bigwedge_{x \in X}\left(A(x) \rightarrow \mathcal{K}\left(\top_{x}\right)(y)\right) \\
\mathcal{K}^{-1}(A)(y) & =\mathcal{K}^{-1}\left(\bigvee_{x \in X}\left(A(x) \odot \top_{x}\right)\right)(y)=\bigwedge_{x \in X}\left(A(x) \rightarrow \mathcal{K}^{-1}\left(\top_{x}\right)(y)\right) \\
& =\bigvee_{x \in X}\left(A(x) \rightarrow \mathcal{K}\left(\top_{y}\right)(x)\right) .
\end{aligned}
$$

(2) For $A=\bigwedge_{x \in X}\left(A^{*}(x) \rightarrow \top_{x}^{*}\right)$, by Theorem 9(2), we have

$$
\begin{aligned}
\mathcal{M}(A)(y) & =\mathcal{M}\left(\bigwedge_{x \in X}\left(A^{*}(x) \rightarrow \top^{*}\right)\right)(y)=\bigvee_{x \in X}\left(A^{*}(x) \odot \mathcal{M}\left(\top_{x}^{*}\right)(y)\right) \\
\mathcal{M}^{-1}(A)(y) & =\bigvee_{x \in X}\left(A^{*}(x) \odot \mathcal{M}^{-1}\left(\top_{x}^{*}\right)(y)\right)
\end{aligned}
$$

(3) $\mathcal{M}(\top)(y)=\bigvee_{x}\left(T^{*}(x) \odot \mathcal{M}\left(\top_{x}^{*}\right)\right)=\perp$ and other cases are similarly proved.

(4) By Lemma 10 (11), we have

$$
\begin{aligned}
& \left(\mathcal{K}\left(A^{*}\right)(y)\right)^{*}=\left(\bigwedge_{x \in X}\left(A^{*}(x) \rightarrow \mathcal{K}\left(\top_{x}\right)(y)\right)^{*}\right. \\
& =\bigvee_{x \in X}\left(A^{*}(x) \odot \mathcal{K}^{*}\left(\top_{x}\right)(y)\right)=\bigvee_{x \in X}\left(A^{*}(x) \odot \mathcal{M}\left(\top_{x}^{*}\right)(y)\right) \\
& =\mathcal{M}\left(\bigwedge_{x \in X}\left(A^{*}(x) \rightarrow \top_{x}^{*}\right)(y)=\mathcal{M}(A)(y)\right.
\end{aligned}
$$

$\left(\mathcal{M}\left(A^{*}\right)(y)\right)^{*}=\left(\bigvee_{x \in X}\left(A(x) \odot \mathcal{M}\left(\top_{x}^{*}\right)(y)\right)\right)^{*}=\bigwedge_{x \in X}\left(A(x) \rightarrow \mathcal{M}^{*}\left(\top_{x}^{*}\right)(y)\right)$ $=\bigwedge_{x \in X}\left(A(x) \rightarrow \mathcal{K}\left(\top_{x}\right)(y)\right)=\mathcal{K}(A)(y)$.

(5) Since $\alpha \odot(\alpha \rightarrow A(x)) \odot\left(A(x) \rightarrow \mathcal{K}\left(\top_{x}\right)(y)\right) \leq A(x) \odot(A(x) \rightarrow$ $\left.\mathcal{K}\left(\top_{x}\right)(y)\right) \leq \mathcal{K}\left(\top_{x}\right)(y)$ iff $\alpha \odot\left(A(x) \rightarrow \mathcal{K}\left(\top_{x}\right)(y)\right) \leq(\alpha \rightarrow A(x)) \rightarrow \mathcal{K}\left(\top_{x}\right)(y)$, then $\alpha \odot \mathcal{K}(A) \leq \mathcal{K}(\alpha \rightarrow A)$.

Since $\alpha \odot\left(\alpha \rightarrow A^{*}\right) \odot \mathcal{M}\left(\top_{x}^{*}\right)(y) \leq A^{*} \odot \mathcal{M}\left(\top_{x}^{*}\right)(y)$ iff $\left(\alpha \rightarrow A^{*}\right) \odot$ $\mathcal{M}\left(\top_{x}^{*}\right)(y) \leq \alpha \rightarrow A^{*} \odot \mathcal{M}\left(\top_{x}^{*}\right)(y)$, then $\mathcal{M}(\alpha \odot A) \leq \alpha \rightarrow \mathcal{M}(A)$.

(6) By $(4), \mathcal{M}\left(\top_{x} \rightarrow \alpha\right)(z)=\mathcal{K}^{*}\left(\top_{x} \odot \alpha^{*}\right)(z)$ and

$$
\begin{aligned}
& \mathcal{M}\left(\top_{x} \rightarrow \alpha\right)(z)=\bigvee_{y \in X}\left(\mathcal{M}\left(\top_{y}^{*}\right)(z) \odot\left(\top_{x} \rightarrow \alpha\right)^{*}(y)\right) \\
& =\bigvee_{y \in X}\left(\mathcal{M}\left(\top_{y}^{*}\right)(z) \odot\left(\top_{x} \odot \alpha^{*}\right)(y)\right)=\mathcal{M}\left(\top_{x}^{*}\right)(z) \odot \alpha^{*} \\
& =\mathcal{K}^{*}\left(\top_{x}\right)(z) \odot \alpha^{*}=\left(\alpha^{*} \rightarrow \mathcal{K}\left(\top_{x}\right)(z)\right)^{*}=\mathcal{K}^{*}\left(\top_{x} \odot \alpha^{*}\right)(z)
\end{aligned}
$$

(7) Since $A(y) \odot(A(y) \rightarrow \alpha) \leq \alpha$ iff $A(y) \leq(A(y) \rightarrow \alpha) \rightarrow \alpha$, we have

$$
\bigwedge_{\alpha \in L}((A(y) \rightarrow \alpha) \rightarrow \alpha) \geq A(y) .
$$

Put $\alpha=A(y)$. Then $\left.\bigwedge_{\alpha \in L}((A(y) \rightarrow \alpha) \rightarrow \alpha) \leq(A(y) \rightarrow A(y)) \rightarrow A(y)\right)=$ $A(y)$. Hence $\bigwedge_{\alpha \in L}((A(y) \rightarrow \alpha) \rightarrow \alpha)=A(y)$. 
(8)

$$
\begin{aligned}
& \bigwedge_{\alpha \in L}\left(\mathcal{M}\left(A \odot \alpha^{*}\right)(x) \rightarrow \alpha\right) \\
& =\bigwedge_{\alpha \in L}\left(\bigvee_{x \in X}\left(\mathcal{M}\left(\top_{x}^{*}\right)(y) \odot(A \rightarrow \alpha)(x)\right) \rightarrow \alpha\right) \\
& =\bigwedge_{\alpha \in L} \bigwedge_{x \in X}\left(\mathcal{M}\left(\top_{x}^{*}\right)(y) \rightarrow((A(x) \rightarrow \alpha) \rightarrow \alpha)\right) \\
& =\bigwedge_{x \in X}\left(\mathcal{K}^{*}\left(\top_{x}\right)(y) \rightarrow \bigwedge_{\alpha \in L}((A(x) \rightarrow \alpha) \rightarrow \alpha)\right) \\
& =\bigwedge_{x \in X}\left(\mathcal{K}^{*}\left(\top_{x}\right)(y) \rightarrow A(x)\right)=\bigwedge_{x \in X}\left(A^{*}(x) \rightarrow \mathcal{K}\left(\top_{x}\right)(y)\right) \\
& =\mathcal{K}\left(A^{*}\right)(x)
\end{aligned}
$$

(9)

$$
\begin{aligned}
\alpha \rightarrow \mathcal{K}(A)(z) & =\mathcal{K}(\alpha \odot A)(z) \\
& =\bigwedge_{x \in X}\left((\alpha \odot A)(x) \rightarrow \mathcal{K}\left(\top_{x}\right)(z)\right) \\
& =\bigwedge_{x \in X}\left(\mathcal{K}^{*}\left(\top_{x}\right)(z) \rightarrow(\alpha \odot A)^{*}(x)\right) \\
& =\bigvee_{x \in X}\left(\mathcal{K}^{*}\left(\top_{x}\right)(z) \odot A^{*}(x)\right) \rightarrow \alpha^{*} \\
& =\bigvee_{x \in X}\left(\mathcal{M}\left(\top_{x}^{*}\right)(z) \odot A(x)\right) \rightarrow \alpha^{*} \\
& =\mathcal{M}\left(A^{*}\right)(z) \rightarrow \alpha^{*}
\end{aligned}
$$

(10) Since $\left(A(x) \rightarrow \alpha^{*}\right) \odot \mathcal{M}\left(\top_{x}\right)(y) \odot\left(\mathcal{M}\left(\top_{x}\right)(y) \rightarrow A(x)\right) \leq(A(x) \rightarrow$ $\left.\alpha^{*}\right) \odot A(x) \leq \alpha^{*}$ iff $\left(A(x) \rightarrow \alpha^{*}\right) \odot \mathcal{M}\left(\top_{x}\right)(y) \leq\left(\mathcal{M}\left(\top_{x}\right)(y) \rightarrow A(x)\right) \rightarrow \alpha^{*}$, we have

$$
\begin{aligned}
& \mathcal{M}(A \odot \alpha)=\bigvee_{x \in X}\left((A \odot \alpha)^{*}(x) \odot \mathcal{M}\left(\top_{x}^{*}\right)(y)\right) \\
& =\bigvee_{x \in X}\left(\left(A(x) \rightarrow \alpha^{*}\right) \odot \mathcal{M}\left(\mathrm{T}_{x}^{*}\right)(y)\right) \leq \bigvee_{x \in X}\left(\left(\mathcal{M}\left(\top_{x}^{*}\right)(y) \rightarrow A(x)\right) \rightarrow \alpha^{*}\right) \\
& =\bigvee_{x \in X}\left(\left(\mathcal{K}^{*}\left(\top_{x}\right)(y) \rightarrow A(x)\right) \rightarrow \alpha^{*}\right) \leq\left(\bigwedge_{x \in X}\left(\mathcal{K}^{*}\left(\top_{x}\right)(y) \rightarrow A(x)\right) \rightarrow \alpha^{*}\right. \\
& =\left(\bigwedge_{x \in X}\left(A^{*}(x) \rightarrow \mathcal{K}\left(\top_{x}\right)(y)\right)\right) \rightarrow \alpha=\mathcal{K}\left(A^{*}\right) \rightarrow \alpha^{*} .
\end{aligned}
$$

(11) By Lemma 10 (12), we have

$$
\begin{aligned}
e_{L^{X}}(B, \mathcal{K}(A)) & =\bigwedge_{y \in X}(B(y) \rightarrow \mathcal{K}(A)(y)) \\
& =\bigwedge_{y \in X}\left(B(y) \rightarrow \bigwedge_{x \in X}\left(A(x) \rightarrow \mathcal{K}\left(\top_{x}\right)(y)\right)\right) \\
& =\bigwedge_{y \in X} \bigwedge_{x \in X}\left(B(y) \rightarrow\left(A(x) \rightarrow \mathcal{K}\left(\top_{x}\right)(y)\right)\right) \\
& =\bigwedge_{x \in X}\left(A(x) \rightarrow \bigwedge_{y \in X}\left(B(y) \rightarrow \mathcal{K}\left(\top_{x}\right)(y)\right)\right) \\
& =e_{L^{X}}\left(A, \mathcal{K}^{-1}(B)\right) . \\
e_{L^{X}}(\mathcal{M}(A), B) & =\bigwedge_{y \in X}(\mathcal{M}(A)(y) \rightarrow B(y)) \\
& =\bigwedge_{y \in X}\left(\bigvee_{x \in X}\left(A^{*}(x) \odot \mathcal{M}\left(\top_{x}^{*}\right)(y)\right) \rightarrow B(y)\right) \\
& =\bigwedge_{y \in X} \bigwedge_{x \in X}\left(\mathcal{M}\left(\top_{x}^{*}\right)(y) \rightarrow\left(A^{*}(x) \rightarrow B(y)\right)\right) \\
& =\bigwedge_{y \in X} \bigwedge_{x \in X}\left(\mathcal{M}\left(\top_{x}^{*}\right)(y) \rightarrow\left(B^{*}(y) \rightarrow A(x)\right)\right) \\
& =\bigwedge_{x \in X}\left(\bigvee_{y \in X}\left(B^{*}(y) \odot \mathcal{M}\left(\top_{x}^{*}\right)(y)\right) \rightarrow A(x)\right) \\
& \left.=\bigwedge_{x \in X}\left(\mathcal{M}^{-1}(B)(y)\right) \rightarrow A(x)\right) \\
& =e_{L^{X}}\left(\mathcal{M}^{-1}(B), A\right) .
\end{aligned}
$$


$(12)$

$$
\begin{aligned}
e_{L^{X}}(B, \mathcal{K}(A)) & =\bigwedge_{y \in X}(B(y) \rightarrow \mathcal{K}(A)(y)) \\
& =\bigwedge_{y \in X}\left(B(y) \rightarrow \bigwedge_{x \in X}\left(A(x) \rightarrow \mathcal{K}\left(\top_{x}\right)(y)\right)\right) \\
& =\bigwedge_{y \in X} \bigwedge_{x \in X}\left(B(y) \rightarrow\left(\mathcal{K}^{*}\left(\top_{x}\right)(y) \rightarrow A^{*}(x)\right)\right) \\
& =\bigwedge_{x \in X}\left(\bigvee_{y \in X}\left(B(y) \odot \mathcal{K}^{*}\left(\top_{x}\right)(y)\right) \rightarrow A^{*}(x)\right) \\
& =\bigwedge_{x \in X}\left(\bigvee_{y \in X}\left(B(y) \odot \mathcal{M}^{-1}\left(\top_{y}\right)(x)\right) \rightarrow A^{*}(x)\right) \\
& =e_{L^{X}}\left(\mathcal{M}^{-1}\left(B^{*}\right), A^{*}\right) . \\
e_{L^{X}}(\mathcal{M}(A), B) & =\bigwedge_{y \in X}(\mathcal{M}(A)(y) \rightarrow B(y)) \\
& =\bigwedge_{y \in X}\left(\bigvee_{x \in X}\left(A^{*}(x) \odot \mathcal{M}\left(\top_{x}^{*}\right)(y)\right) \rightarrow B(y)\right) \\
& =\bigwedge_{y \in X} \bigwedge_{x \in X}\left(A^{*}(x) \rightarrow\left(\mathcal{M}\left(\top_{x}^{*}\right)(y) \rightarrow B(y)\right)\right) \\
& =\bigwedge_{y \in X} \bigwedge_{x \in X}\left(A^{*}(x) \rightarrow\left(B^{*}(y) \rightarrow \mathcal{M}^{*}\left(\top_{x}^{*}\right)(y)\right)\right) \\
& =\bigwedge_{x \in X}\left(A^{*}(x) \rightarrow \bigwedge_{y \in X}\left(B^{*}(y) \rightarrow \mathcal{K}\left(\top_{x}\right)(y)\right)\right) \\
& =e_{L^{X}}\left(A^{*}, \mathcal{K}^{-1}(B)\right) .
\end{aligned}
$$

$$
\begin{aligned}
& \mathcal{K}(A)(y) \odot e_{L^{X}}(B, A)=\bigwedge_{x}\left(A(x) \rightarrow \mathcal{K}\left(\top_{x}\right)(y)\right) \odot \bigwedge_{z \in X}(B(z) \rightarrow A(z)) \\
& \leq \bigwedge_{x \in X}\left(\left(A(x) \rightarrow \mathcal{K}\left(\top_{x}\right)(y)\right) \odot(B(x) \rightarrow A(x))\right. \\
& \leq \bigwedge_{x \in X}\left(\left(B(x) \rightarrow \mathcal{K}\left(\top_{x}\right)(y)\right)=\mathcal{K}(B)(y) .\right.
\end{aligned}
$$

Thus, $e_{L^{X}}(B, A) \leq \mathcal{K}(A)(y) \rightarrow \mathcal{K}(B)(y)$. Hence $e_{L^{X}}(B, A) \leq e_{L^{X}}(\mathcal{K}(A), \mathcal{K}(B))$.

$$
\begin{aligned}
& \left.\mathcal{M}^{*}\left(\top_{x}^{*}\right)(y) \odot A^{*}(x)\right) \odot e_{L^{x}}(B, A) \\
& \left.\leq \mathcal{M}^{*}\left(\top_{x}^{*}\right)(y) \odot A^{*}(x)\right) \odot(B(x) \rightarrow A(x)) \\
& \left.=\mathcal{M}^{*}\left(\top_{x}^{*}\right)(y) \odot A^{*}(x)\right) \odot\left(A^{*}(x) \rightarrow B^{*}\right) \leq \mathcal{M}^{*}\left(\top_{x}^{*}\right)(y) \odot B^{*}(x) .
\end{aligned}
$$

Thus, $\left.e_{L^{X}}(B, A) \leq \mathcal{M}^{*}\left(\top_{x}^{*}\right)(y) \odot A^{*}(x)\right) \rightarrow \mathcal{M}^{*}\left(\top_{x}^{*}\right)(y) \odot B^{*}(x)$. Hence

$$
e_{L^{X}}(A, B) \leq e_{L^{X}}(\mathcal{M}(B), \mathcal{M}(A)) .
$$

Theorem 12. Let $\left(L^{X}, e_{L^{X}}\right)$ be a fuzzy poset. Let $\mathcal{K}, \mathcal{K}^{-1}: L^{X} \rightarrow$ $L^{X}$ be join-meet preserving maps such that $\mathcal{K}^{-1}\left(\top_{x}\right)(y)=\mathcal{K}\left(\top_{y}\right)(x)$ for all $x, y \in X$. Let $\mathcal{M}, \mathcal{M}^{-1}: L^{X} \rightarrow L^{X}$ be meet-join preserving maps such that $\mathcal{M}^{-1}\left(\top_{x}^{*}\right)(y)=\mathcal{M}\left(\top_{y}^{*}\right)(x)$ for all $x, y \in X$. For $x, y, z \in X$ and $A \in L^{X}$, we have the following properties.

(1) If $\top_{x} \leq \mathcal{M}\left(\top_{x}^{*}\right)$, then $A^{*} \leq \mathcal{M}(A)$ and $A^{*} \leq \mathcal{M}^{-1}(A)$. 
(2) If $\mathcal{K}\left(\top_{x}\right) \leq \top_{x}^{*}$, then $\mathcal{K}(A) \leq A^{*}$ and $\mathcal{K}^{-1}(A) \leq A^{*}$.

(3) $\bigvee_{y \in X} \mathcal{K}^{*}\left(\top_{x}\right)(y) \odot \mathcal{K}^{*}\left(\top_{y}\right)(z) \leq \mathcal{K}^{*}\left(\top_{x}\right)(z)$ for all $x, z \in X$ iff $\mathcal{K}\left(\mathcal{K}^{*}\left(\top_{x}\right)\right)$ $\geq \mathcal{K}\left(\top_{x}\right)$ iff $\mathcal{K}^{-1}\left(\mathcal{K}\left(\top_{x}\right)\right) \geq \mathcal{K}^{*}\left(\top_{x}\right)$ iff $\mathcal{K}\left(\mathcal{K}^{*}(A)\right) \geq \mathcal{K}(A)$ iff $\mathcal{K}^{-1}(\mathcal{K}(A)) \geq$ $\mathcal{K}^{*}(A)$.

(4) $\bigvee_{x \in X} \mathcal{K}^{*}\left(\top_{x}\right)(y) \odot \mathcal{K}^{*}\left(\top_{x}\right)(z) \leq \mathcal{K}^{*}\left(\top_{y}\right)(z)$ for all $x, z \in X$ iff $\mathcal{K}\left(\mathcal{K}^{-1 *}\left(\top_{y}\right)\right)$ $\geq \mathcal{K}\left(\top_{y}\right)$ iff $\mathcal{K}^{-1}\left(\mathcal{K}\left(\top_{y}\right)\right) \geq \mathcal{K}^{-1 *}\left(\top_{y}\right)$ iff $\mathcal{K}\left(\mathcal{K}^{-1 *}(A)\right) \leq \mathcal{K}(A)$ iff $\mathcal{K}^{-1}(\mathcal{K}(A)) \leq$ $\mathcal{K}^{-1 *}(A)$.

(5) $\bigvee_{x \in X} \mathcal{K}\left(\top_{y}\right)(x) \odot \mathcal{K}\left(\top_{z}\right)(x) \leq \mathcal{K}\left(\top_{y}\right)(z)$ for all $x, z \in X$ iff $\mathcal{K}^{-1}\left(\mathcal{K}^{*}\left(\top_{y}\right)\right)$ $\geq \mathcal{K}\left(\top_{y}\right)$ iff $\mathcal{K}\left(\mathcal{K}\left(\top_{y}\right)\right) \geq \mathcal{K}^{*}\left(\top_{y}\right)$ iff $\mathcal{K}^{-1}\left(\mathcal{K}^{*}(A)\right) \geq \mathcal{K}(A)$ iff $\mathcal{K}(\mathcal{K}(A)) \geq \mathcal{K}^{*}(A)$.

(6) $\bigvee_{y \in X} \mathcal{M}\left(\mathrm{T}_{x}^{*}\right)(y) \odot \mathcal{M}\left(\mathrm{T}_{y}^{*}\right)(z) \leq \mathcal{M}\left(\mathrm{T}_{x}^{*}\right)(z)$ for all $y, z \in X$ iff $\mathcal{M}\left(\mathcal{M}^{*}\left(\mathrm{~T}_{x}^{*}\right)\right)$ $\leq \mathcal{M}\left(\top_{x}^{*}\right)$ iff $\mathcal{M}^{-1}\left(\mathcal{M}^{-1 *}\left(\top_{x}^{*}\right)\right) \leq \mathcal{M}^{-1}\left(\top_{x}^{*}\right)$ iff $\mathcal{M}\left(\mathcal{M}^{*}(A)\right) \leq \mathcal{M}(A)$ iff $\mathcal{M}^{-1}\left(\mathcal{M}^{-1 *}(A)\right) \leq \mathcal{M}^{-1}(A)$.

(7) $\bigvee_{x \in X} \mathcal{M}\left(\top_{x}^{*}\right)(y) \odot \mathcal{M}\left(\top_{x}^{*}\right)(z) \leq \mathcal{M}\left(\top_{y}^{*}\right)(z)$ for all $x, y \in X$ iff $\mathcal{M}\left(\mathcal{M}^{-1 *}\left(\top_{x}^{*}\right)\right) \leq \mathcal{M}\left(\top_{x}^{*}\right)$ iff $\mathcal{M}\left(\mathcal{M}^{-1 *}\left(\top_{z}^{*}\right)\right) \leq \mathcal{M}^{-1}\left(\top_{z}^{*}\right)$ iff $\mathcal{M}\left(\mathcal{M}^{-1 *}(A)\right) \leq$ $\mathcal{M}(A)$ iff $\mathcal{M}\left(\mathcal{M}^{-1 *}(A)\right) \leq \mathcal{M}^{-1}(A)$.

(8) $\bigvee_{x \in X} \mathcal{M}\left(\top_{y}^{*}\right)(x) \odot \mathcal{M}\left(\top_{z}^{*}\right)(x) \leq \mathcal{M}\left(\top_{y}^{*}\right)(z)$ for all $y, z \in X$ iff $\mathcal{M}^{-1}\left(\mathcal{M}^{*}\left(\top_{z}^{*}\right)\right) \leq \mathcal{M}^{-1}\left(\top_{z}^{*}\right)$ iff $\mathcal{M}^{-1}\left(\mathcal{M}^{*}\left(\top_{y}^{*}\right)\right) \leq \mathcal{M}\left(\top_{y}^{*}\right)$ iff $\mathcal{M}^{-1}\left(\mathcal{M}^{*}(A)\right) \leq$ $\mathcal{M}^{-1}(A)$ iff $\mathcal{M}^{-1}\left(\mathcal{M}^{*}(A)\right) \leq \mathcal{M}(A)$.

(9) If $\mathcal{K}^{*}\left(\top_{x}\right)(y)=\mathcal{M}\left(\top_{x}^{*}\right)(y)$ for all $x, y \in X$, then $\bigvee_{y \in X} \mathcal{K}^{*}\left(\top_{x}\right)(y) \odot$ $\mathcal{K}^{*}\left(\top_{y}\right)(z) \leq \mathcal{K}^{*}\left(\top_{x}\right)(z)$ for all $x, z \in X$ iff $\mathcal{M}\left(\mathcal{K}\left(\top_{x}\right)\right) \leq \mathcal{K}^{*}\left(\top_{x}\right)$ iff $\mathcal{M}^{-1}\left(\mathcal{K}^{-1}\left(\top_{z}\right)\right) \leq \mathcal{K}^{-1 *}\left(\top_{z}\right)$ iff $\mathcal{M}(\mathcal{K}(A)) \leq \mathcal{K}^{*}(A)$ iff $\mathcal{M}^{-1}\left(\mathcal{K}^{-1}(A)\right) \leq \mathcal{K}^{-1 *}(A)$.

(10) If $\mathcal{K}^{*}\left(\top_{x}\right)(y)=\mathcal{M}\left(\top_{x}^{*}\right)(y)$ for all $x, y \in X$, then $\bigvee_{x \in X} \mathcal{K}^{*}\left(\top_{x}\right)(y) \odot$ $\mathcal{K}^{*}\left(\top_{x}\right)(z) \leq \mathcal{K}^{*}\left(\top_{y}\right)(z)$ for all $x, z \quad \underset{\in}{\in} \quad X \quad$ iff $\mathcal{M}\left(\mathcal{K}^{-1}\left(\top_{y}\right)\right) \leq \mathcal{K}^{*}\left(\top_{y}\right)$ iff $\mathcal{M}\left(\mathcal{K}^{-1}\left(\top_{z}\right)\right) \leq \mathcal{K}^{-1 *}\left(\top_{z}\right)$ iff $\mathcal{M}\left(\mathcal{K}^{-1}(A)\right) \leq \mathcal{K}^{*}(A)$ iff $\mathcal{M}\left(\mathcal{K}^{-1}(A)\right) \leq \mathcal{K}^{-1 *}(A)$.

(11) If $\mathcal{K}^{*}\left(\top_{x}\right)(y)=\mathcal{M}\left(\top_{x}^{*}\right)(y)$ for all $x, y \in X$, then $\bigvee_{x \in X} \mathcal{K}^{*}\left(\top_{y}\right)(x) \odot$ $\mathcal{K}^{*}\left(\top_{z}\right)(x) \leq \mathcal{K}^{*}\left(\top_{y}\right)(z)$ for all $x, z \in X$ iff $\mathcal{M}^{-1}\left(\mathcal{K}\left(\top_{y}\right)\right) \leq \mathcal{K}^{*}\left(\top_{y}\right)$ iff $\mathcal{M}^{-1}\left(\mathcal{K}\left(\top_{z}\right)\right) \leq \mathcal{K}^{-1 *}\left(\top_{z}\right)$ iff $\mathcal{M}^{-1}(\mathcal{K}(A)) \leq \mathcal{K}^{*}(A)$ iff $\mathcal{M}^{-1}(\mathcal{K}(A)) \leq \mathcal{K}^{-1 *}(A)$.

Proof. (1) For $A=\bigwedge_{x \in X}\left(A^{*}(x) \rightarrow \top_{x}^{*}\right)$, we have

$$
\begin{aligned}
\mathcal{M}(A)(y) & =\mathcal{M}\left(\bigwedge_{x \in X}\left(A^{*}(x) \rightarrow \top_{x}^{*}\right)\right)(y)=\bigvee_{x \in X}\left(A^{*}(x) \odot \mathcal{M}\left(\top_{x}^{*}\right)(y)\right) \\
& \geq \bigvee_{x \in X}\left(A^{*}(x) \odot \top_{x}^{*}(y)\right)=A^{*}(y) .
\end{aligned}
$$

Similarly, we have $\mathcal{M}^{-1}(A) \geq A^{*}$ for each $A \in L^{X}$.

(2) For $A=\bigvee_{x \in X}\left(A(x) \odot \top_{x}\right)$, we have

$$
\begin{aligned}
\mathcal{K}(A)(y) & =\mathcal{K}\left(\bigvee_{x \in X}\left(A(x) \odot \top_{x}\right)\right)(y)=\bigwedge_{x \in X}\left(A(x) \rightarrow \mathcal{K}\left(\top_{x}\right)(y)\right) \\
& \leq \bigwedge_{x \in X}\left(A(x) \rightarrow \top_{x}^{*}(y)\right)=A^{*}(y) .
\end{aligned}
$$

Similarly, we have $\mathcal{K}^{-1}(A) \geq A^{*}$ for each $A \in L^{X}$. 
(3) Since $\bigvee_{y \in X} \mathcal{K}^{*}\left(\top_{x}\right)(y) \odot \mathcal{K}^{*}\left(\top_{y}\right)(z) \leq \mathcal{K}^{*}\left(\top_{x}\right)(z)$ for all $x, z \in X$, then $\bigwedge_{y \in X} \mathcal{K}^{*}\left(\top_{x}\right)(y) \rightarrow \mathcal{K}\left(\top_{y}\right)(z)=\mathcal{K}\left(\bigvee_{y \in X} \mathcal{K}^{*}\left(\top_{x}\right)(y) \odot \top_{y}\right)=\mathcal{K}\left(\mathcal{K}^{*}\left(\top_{x}\right)\right)(z) \geq$ $\mathcal{K}\left(\top_{x}\right)(z)$ for all $x, z \in X$.

Since $\bigvee_{y \in X} \mathcal{K}^{*}\left(\top_{x}\right)(y) \odot \mathcal{K}^{*}\left(\top_{y}\right)(z) \leq \mathcal{K}^{*}\left(\top_{x}\right)(z)$ for all $x, z \in X$, then $\mathcal{K}^{*}\left(\top_{x}\right)(y) \leq \mathcal{K}^{*}\left(\top_{y}\right)(z) \rightarrow \mathcal{K}^{*}\left(\top_{x}\right)(z)$.

$\mathcal{K}^{*}\left(\top_{x}\right)(y) \leq \bigwedge_{y \in X}\left(\mathcal{K}^{*}\left(\top_{y}\right)(z) \rightarrow \mathcal{K}^{*}\left(\top_{x}\right)(z)\right)=\bigwedge_{y \in X}\left(\mathcal{K}\left(\top_{x}\right)(z) \rightarrow\right.$ $\left.\mathcal{K}\left(\top_{y}\right)(z)\right)=\bigwedge_{y \in X}\left(\mathcal{K}\left(\top_{x}\right)(z) \rightarrow \mathcal{K}^{-1}\left(\top_{z}\right)(y)\right)=\mathcal{K}^{-1}\left(\mathcal{K}\left(\top_{x}\right)\right)(y)$.

$$
\begin{aligned}
\mathcal{K}\left(\mathcal{K}^{*}(A)\right)(z) & =\mathcal{K}\left(\bigvee_{y \in X}\left(\mathcal{K}^{*}(A)(y) \odot \top_{y}\right)(z)=\bigwedge_{y \in X}\left(\mathcal{K}^{*}(A)(y) \rightarrow \mathcal{K}\left(\top_{x}\right)(z)\right.\right. \\
& =\bigwedge_{y \in X}\left(\left(\bigwedge_{x \in X}\left(A(x) \rightarrow \mathcal{K}\left(\top_{x}\right)(y)\right)\right)^{*} \rightarrow \mathcal{K}\left(\top_{y}\right)(z)\right. \\
& =\bigwedge_{y \in X}\left(\bigvee_{x \in X}\left(A(x) \odot \mathcal{K}^{*}\left(\top_{x}\right)(y)\right) \rightarrow \mathcal{K}\left(\top_{y}\right)(z)\right. \\
& =\bigwedge_{y \in X} \bigwedge_{x \in X}\left(\mathcal{K}^{*}\left(\top_{x}\right)(y) \odot \mathcal{K}^{*}\left(\top_{y}\right)(z) \rightarrow A^{*}(x)\right) \\
& \geq \bigwedge_{y \in X}\left(\mathcal{K}^{*}\left(\top_{x}\right)(z) \rightarrow A^{*}(x)\right) \\
& =\mathcal{K}(A)(z) .
\end{aligned}
$$

$$
\begin{aligned}
\mathcal{K}^{-1}(\mathcal{K}(A))(z)= & \mathcal{K}^{-1}\left(\bigvee_{y \in X}\left(\mathcal{K}(A)(y) \odot \top_{y}\right)(z)=\bigwedge_{y \in X}(\mathcal{K}(A)(y)\right. \\
& \left.\rightarrow \mathcal{K}^{-1}\left(\top_{y}\right)(z)\right) \\
= & \bigwedge_{y \in X}\left(\left(\bigwedge_{x \in X}\left(A(x) \rightarrow \mathcal{K}\left(\top_{x}\right)(y)\right)\right) \rightarrow \mathcal{K}^{-1}\left(\top_{y}\right)(z)\right. \\
= & \bigwedge_{y \in X}\left(\mathcal{K}^{-1 *}\left(\top_{y}\right)(z) \rightarrow\left(\bigvee_{x \in X}\left(A(x) \odot \mathcal{K}^{*}\left(\top_{x}\right)(y)\right)\right)\right)
\end{aligned}
$$

Since

$$
\begin{aligned}
& \mathcal{K}^{*}\left(\top_{x}\right)(z) \odot \mathcal{K}^{*}\left(\top_{z}\right)(y) \odot\left(\mathcal{K}^{*}\left(\top_{x}\right)(y) \rightarrow A^{*}(x)\right) \\
& \leq \mathcal{K}^{*}\left(\top_{x}\right)(y) \odot\left(\mathcal{K}^{*}\left(\top_{x}\right)(y) \rightarrow A^{*}(x)\right) \leq A^{*}(x) \\
& \text { iff } \mathcal{K}^{*}\left(\top_{z}\right)(y) \odot\left(\mathcal{K}^{*}\left(\top_{x}\right)(y) \rightarrow A^{*}(x)\right) \leq \mathcal{K}^{*}\left(\top_{x}\right)(z) \rightarrow A^{*}(x) \\
&\left(\mathcal{K}^{-1}(\mathcal{K}(A))(z)\right)^{*}=\left(\bigwedge_{y \in X}\left(\mathcal{K}^{-1 *}\left(\top_{y}\right)(z) \rightarrow\left(\bigvee_{x \in X}\left(A(x) \odot \mathcal{K}^{*}\left(\top_{x}\right)(y)\right)\right)\right)\right)^{*} \\
&=\bigvee_{y \in X}\left(\mathcal{K}^{-1 *}\left(\top_{y}\right)(z) \odot \bigwedge_{x \in X}\left(\mathcal{K}^{*}\left(\top_{x}\right)(y) \rightarrow A^{*}(x)\right)\right) \\
& \leq \bigvee_{y \in X} \bigvee_{x \in X}\left(\mathcal{K}^{-1 *}\left(\top_{y}\right)(z) \odot\left(\mathcal{K}^{*}\left(\top_{x}\right)(y) \rightarrow A^{*}(x)\right)\right) \\
& \leq \bigvee_{x \in X}\left(\mathcal{K}^{*}\left(\top_{x}\right)(z) \rightarrow A^{*}(x)\right)=\mathcal{K}(A)(z)
\end{aligned}
$$

Other cases are similarly proved.

(4) Since $\bigvee_{x \in X} \mathcal{K}^{*}\left(\top_{x}\right)(y) \odot \mathcal{K}^{*}\left(\top_{x}\right)(z) \leq \mathcal{K}^{*}\left(\top_{x}\right)(z)$ for all $x, z \in X$, then $\bigwedge_{x \in X} \mathcal{K}^{*}\left(\top_{x}\right)(y) \rightarrow \mathcal{K}\left(\top_{x}\right)(z)=\mathcal{K}\left(\bigvee_{x \in X} \mathcal{K}^{-1 *}\left(\top_{y}\right)(x) \odot \top_{x}\right)(z)=$ $\mathcal{K}\left(\mathcal{K}^{-1 *}\left(\top_{y}\right)\right)(z) \geq \mathcal{K}\left(\top_{y}\right)(z)$ for all $y, z \in X$.

Since $\bigvee_{y \in X} \mathcal{K}^{*}\left(\top_{x}\right)(y) \odot \mathcal{K}^{*}\left(\top_{x}\right)(z) \leq \mathcal{K}^{*}\left(\top_{y}\right)(z)$ for all $x, z \in X$, then $\mathcal{K}^{*}\left(\top_{x}\right)(y) \leq \mathcal{K}^{*}\left(\top_{x}\right)(z) \rightarrow \mathcal{K}^{*}\left(\top_{y}\right)(z)$.

$$
\mathcal{K}^{*}\left(\top_{x}\right)(y) \leq \bigwedge_{z \in X}\left(\mathcal{K}^{*}\left(\top_{x}\right)(z) \rightarrow \mathcal{K}^{*}\left(\top_{y}\right)(z)\right)=\bigwedge_{y \in X}\left(\mathcal{K}\left(\top_{y}\right)(z)\right.
$$




$$
\begin{aligned}
&\left.\rightarrow \mathcal{K}\left(\top_{x}\right)(z)\right)=\bigwedge_{y \in X}\left(\mathcal{K}\left(\top_{y}\right)(z) \rightarrow \mathcal{K}^{-1}\left(\top_{z}\right)(x)\right)=\mathcal{K}^{-1}\left(\mathcal{K}\left(\top_{y}\right)\right)(x) . \\
& \mathcal{K}\left(\mathcal{K}^{-1 *}(A)\right)(z)= \mathcal{K}\left(\bigvee_{y \in X}\left(\mathcal{K}^{-1 *}(A)(y) \odot \top_{y}\right)(z)=\bigwedge_{y \in X}\left(\mathcal{K}^{-1 *}(A)(y)\right.\right. \\
& \rightarrow \mathcal{K}\left(\top_{x}\right)(z) \\
&= \bigwedge_{y \in X}\left(\left(\bigwedge_{x \in X}\left(A(x) \rightarrow \mathcal{K}^{-1}\left(\top_{x}\right)(y)\right)\right)^{*} \rightarrow \mathcal{K}\left(\top_{y}\right)(z)\right. \\
&= \bigwedge_{y \in X}\left(\bigvee_{x \in X}\left(A(x) \odot \mathcal{K}^{-1 *}\left(\top_{x}\right)(y)\right) \rightarrow \mathcal{K}\left(\top_{y}\right)(z)\right. \\
&= \bigwedge_{x \in X} \bigwedge_{y \in X}\left(\mathcal{K}^{*}\left(\top_{y}\right)(x) \odot \mathcal{K}^{*}\left(\top_{y}\right)(z) \rightarrow A^{*}(x)\right) \\
& \geq \bigwedge_{x \in X}\left(\mathcal{K}^{*}\left(\top_{x}\right)(z) \rightarrow A^{*}(x)\right) \\
&= \mathcal{K}(A)(z) . \\
& \\
&= \mathcal{K}^{-1}\left(\bigvee_{y \in X}\left(\mathcal{K}(A)(y) \odot \top_{y}\right)(z)=\bigwedge_{y \in X}(\mathcal{K}(A)(y)\right. \\
&\left.\rightarrow \mathcal{K}^{-1}\left(\top_{y}\right)(z)\right) \\
&= \bigwedge_{y \in X}\left(\left(\bigwedge_{x \in X}\left(A(x) \rightarrow \mathcal{K}\left(\top_{x}\right)(y)\right)\right) \rightarrow \mathcal{K}^{-1}\left(\top_{y}\right)(z)\right. \\
&= \bigwedge_{y \in X}\left(\mathcal{K}^{-1 *}\left(\top_{y}\right)(z) \rightarrow\left(\bigvee_{x \in X}\left(A(x) \odot \mathcal{K}^{*}\left(\top_{x}\right)(y)\right)\right)\right.
\end{aligned}
$$

Since

$$
\begin{aligned}
& \mathcal{K}^{*}\left(\top_{z}\right)(x) \odot \mathcal{K}^{*}\left(\top_{z}\right)(y) \odot\left(\mathcal{K}^{*}\left(\top_{x}\right)(y) \rightarrow A^{*}(x)\right) \leq \mathcal{K}^{*}\left(\top_{x}\right)(y) \odot\left(\mathcal{K}^{*}\left(\top_{x}\right)(y) \rightarrow A^{*}(x)\right) \leq A^{*}(x) \\
& \text { iff } \mathcal{K}^{*}\left(\top_{z}\right)(y) \odot\left(\mathcal{K}^{*}\left(\top_{x}\right)(y) \rightarrow A^{*}(x)\right) \leq \mathcal{K}^{*}\left(\top_{z}\right)(x) \rightarrow A^{*}(x) \\
&\left(\mathcal{K}^{-1}(\mathcal{K}(A))(z)\right)^{*}=\left(\bigwedge_{y \in X}\left(\mathcal{K}^{-1 *}\left(\top_{y}\right)(z) \rightarrow\left(\bigvee_{x \in X}\left(A(x) \odot \mathcal{K}^{*}\left(\top_{x}\right)(y)\right)\right)\right)\right)^{*} \\
&=\bigvee_{y \in X}\left(\mathcal{K}^{-1 *}\left(\top_{y}\right)(z) \odot \bigwedge_{x \in X}\left(\mathcal{K}^{*}\left(\top_{x}\right)(y) \rightarrow A^{*}(x)\right)\right) \\
& \leq \bigvee_{y \in X} \bigvee_{x \in X}\left(\mathcal{K}^{-1 *}\left(\top_{y}\right)(z) \odot\left(\mathcal{K}^{*}\left(\top_{x}\right)(y) \rightarrow A^{*}(x)\right)\right) \\
& \leq \bigvee_{x \in X}\left(\mathcal{K}^{*}\left(\top_{z}\right)(x) \rightarrow A^{*}(x)\right)=\mathcal{K}^{-1}(A)(z)
\end{aligned}
$$

Other cases are similarly proved.

(5) It is similarly proved as (4).

(6) For $\mathcal{M}\left(\top_{x}^{*}\right)=\bigwedge_{y \in X}\left(\mathcal{M}^{*}\left(\top_{x}^{*}\right)(y) \rightarrow \top_{y}^{*}\right)$, we have

$$
\begin{aligned}
& \bigvee_{y \in X} \mathcal{M}\left(\top_{x}^{*}\right)(y) \odot \mathcal{M}\left(\top_{y}^{*}\right)(z) \leq \mathcal{M}\left(\top_{x}^{*}\right)(z) \\
& \quad \operatorname{iff} \mathcal{M}\left(\bigwedge_{y \in X}\left(\mathcal{M}\left(\top_{x}^{*}\right)(y) \rightarrow \top_{y}^{*}\right)(z) \leq \mathcal{M}\left(\top_{x}^{*}\right)(z)\right. \\
& \quad \operatorname{iff} \mathcal{M}\left(\mathcal{M}^{*}\left(\top_{x}^{*}\right)\right)(z) \leq \mathcal{M}\left(\top_{x}^{*}\right)(z) \\
& \bigvee_{y \in X} \mathcal{M}\left(\top_{x}^{*}\right)(y) \odot \mathcal{M}\left(\top_{y}^{*}\right)(z) \leq \mathcal{M}\left(\top_{x}^{*}\right)(z) \\
& \text { iff } \bigvee_{y \in X} \mathcal{M}^{-1}\left(\top_{y}^{*}\right)(x) \odot \mathcal{M}^{-1}\left(\top_{z}^{*}\right)(y) \leq \mathcal{M}\left(\top_{x}^{*}\right)(z) \\
& \text { iff } \mathcal{M}^{-1}\left(\bigwedge_{y \in X}\left(\mathcal{M}^{-1}\left(\top_{z}^{*}\right)(y) \rightarrow \top_{y}^{*}\right)(x) \leq \mathcal{M}^{-1}\left(\top_{z}^{*}\right)(x)\right. \\
& \text { iff } \mathcal{M}^{-1}\left(\mathcal{M}^{-1 *}\left(\top_{z}^{*}\right)\right)(x) \leq \mathcal{M}^{-1}\left(\top_{z}^{*}\right)(x)
\end{aligned}
$$




$$
\begin{aligned}
\mathcal{M}\left(\mathcal{M}^{*}(A)\right)(z) & =\mathcal{M}\left(\bigwedge_{y \in X}\left(\mathcal{M}(A)(y) \rightarrow \top_{y}^{*}\right)\right) \\
& =\bigvee_{y \in X}\left(\mathcal{M}(A)(y) \odot \mathcal{M}\left(\top_{y}^{*}\right)(z)\right) \\
& =\bigvee_{y \in X}\left(\bigvee_{x \in X}\left(A^{*}(x) \odot \mathcal{M}\left(\top_{x}^{*}\right)(y)\right) \odot \mathcal{M}\left(\top_{y}^{*}\right)(z)\right) \\
& \left.\leq \bigvee_{x \in X}\left(A^{*}(x) \odot \mathcal{M}\left(\top_{x}^{*}\right)(z)\right)=\mathcal{M}(A)(z)\right)
\end{aligned}
$$

(7) For $\mathcal{M}\left(\top_{x}^{*}\right)=\bigwedge_{y \in X}\left(\mathcal{M}^{*}\left(\top_{x}^{*}\right)(y) \rightarrow \top_{y}^{*}\right)$, we have

$$
\begin{aligned}
& \quad \bigvee_{y \in X} \mathcal{M}\left(\top_{x}^{*}\right)(y) \odot \mathcal{M}\left(\top_{x}^{*}\right)(z) \leq \mathcal{M}\left(\top_{y}^{*}\right)(z) \\
& \quad \text { iff } \mathcal{M}\left(\bigwedge_{x \in X}\left(\mathcal{M}^{-1}\left(\top_{y}^{*}\right)(z) \rightarrow \top_{x}^{*}\right)(z) \leq \mathcal{M}\left(\top_{y}^{*}\right)(z)\right. \\
& \quad \text { iff } \mathcal{M}\left(\mathcal{M}^{-1 *}\left(\top_{y}^{*}\right)\right)(z) \leq \mathcal{M}\left(\top_{y}^{*}\right)(z) \\
& \bigvee_{x \in X} \mathcal{M}\left(\top_{x}^{*}\right)(y) \odot \mathcal{M}\left(\top_{x}^{*}\right)(z) \leq \mathcal{M}\left(\top_{y}^{*}\right)(z) \\
& \text { iff } \bigvee_{x \in X} \mathcal{M}\left(\top_{x}^{*}\right)(y) \odot \mathcal{M}^{-1}\left(\top_{z}^{*}\right)(x) \leq \mathcal{M}\left(\top_{y}^{*}\right)(z) \\
& \text { iff } \mathcal{M}\left(\bigwedge_{x \in X}\left(\mathcal{M}^{-1}\left(\top_{z}^{*}\right)(x) \rightarrow T_{x}^{*}\right)(y) \leq \mathcal{M}^{-1}\left(\top_{z}^{*}\right)(y)\right. \\
& \text { iff } \mathcal{M}\left(\mathcal{M}^{-1 *}\left(\top_{z}^{*}\right)\right)(y) \leq \mathcal{M}^{-1}\left(\top_{z}^{*}\right)(y)
\end{aligned}
$$

$$
\begin{aligned}
\mathcal{M}\left(\mathcal{M}^{-1 *}(A)\right)(z) & =\mathcal{M}\left(\bigwedge_{y \in X}\left(\mathcal{M}^{-1}(A)(y) \rightarrow \top_{y}^{*}\right)\right) \\
& =\bigvee_{y \in X}\left(\mathcal{M}^{-1}(A)(y) \odot \mathcal{M}\left(\top_{y}^{*}\right)(z)\right) \\
& =\bigvee_{y \in X}\left(\bigvee_{x \in X}\left(A^{*}(x) \odot \mathcal{M}^{-1}\left(\top_{x}^{*}\right)(y)\right) \odot \mathcal{M}\left(\top_{y}^{*}\right)(z)\right) \\
& \left.\leq \bigvee_{x \in X}\left(A^{*}(x) \odot \mathcal{M}\left(\top_{x}^{*}\right)(z)\right)=\mathcal{M}(A)(z)\right)
\end{aligned}
$$

(8) It is similarly proved.

(9) Since $\bigvee_{y \in X} \mathcal{K}^{*}\left(\top_{x}\right)(y) \odot \mathcal{M}\left(\top_{y}^{*}\right)(z)=\mathcal{M}\left(\bigwedge_{y \in X}\left(\mathcal{K}^{*}\left(\top_{x}\right)(y) \rightarrow \top_{y}\right)\right)(z)=$ $\mathcal{M}\left(\mathcal{K}\left(\top_{x}\right)\right)(z)$, we have

$$
\begin{aligned}
& \bigvee_{y \in X} \mathcal{K}^{*}\left(\top_{x}\right)(y) \odot \mathcal{K}^{*}\left(\top_{y}\right)(z) \leq \mathcal{K}^{*}\left(\top_{x}\right)(z) \\
& \text { iff } \bigvee_{y \in X} \mathcal{K}^{*}\left(\top_{x}\right)(y) \odot \mathcal{M}\left(\top_{y}^{*}\right)(z) \leq \mathcal{K}^{*}\left(\top_{x}\right)(z) \\
& \text { iff } \mathcal{M}\left(\mathcal{K}\left(\top_{x}\right)\right)(z) \leq \mathcal{K}^{*}\left(\top_{x}\right)(z) .
\end{aligned}
$$

Since $\bigvee_{y \in X} \mathcal{K}^{-1 *}\left(\top_{z}\right)(y) \odot \mathcal{M}^{-1}\left(\top_{y}^{*}\right)(x)=\mathcal{M}^{-1}\left(\bigwedge_{y \in X}\left(\mathcal{K}^{-1 *}\left(\top_{z}\right)(y) \rightarrow \top_{y}^{*}\right)\right)(x)=$ $\mathcal{M}^{-1}\left(\mathcal{K}^{-1}\left(\top_{z}\right)\right)(x)$, we have

$$
\begin{aligned}
& \bigvee_{y \in X} \mathcal{K}^{*}\left(\top_{x}\right)(y) \odot \mathcal{K}^{*}\left(\top_{y}\right)(z) \leq \mathcal{K}^{*}\left(\top_{x}\right)(z) \\
& \text { iff } \bigvee_{y \in X} \mathcal{K}^{-1 *}\left(\top_{z}\right)(y) \odot \mathcal{K}^{-1 *}\left(\top_{y}\right)(x) \leq \mathcal{K}^{*}\left(\top_{x}\right)(z) \\
& \text { iff } \bigvee_{y \in X} \mathcal{K}^{*}\left(\top_{x}\right)(y) \odot \mathcal{M}\left(\top_{y}^{*}\right)(z) \leq \mathcal{K}^{-1 *}\left(\top_{z}\right)(x) \\
& \text { iff } \mathcal{M}^{-1}\left(\mathcal{K}^{-1}\left(\top_{z}\right)\right)(x) \leq \mathcal{K}^{-1 *}\left(\top_{z}\right)(x) .
\end{aligned}
$$




$$
\begin{aligned}
\mathcal{M}(\mathcal{K}(A))(z) & =\mathcal{M}\left(\bigwedge_{y \in X}\left(\mathcal{K}^{*}(A)(y) \rightarrow \top_{y}^{*}\right)\right)(z) \\
& =\bigvee_{y \in X}\left(\mathcal{K}^{*}(A)(y) \odot \mathcal{M}\left(\top_{y}^{*}\right)(z)\right) \\
& =\bigvee_{y \in X}\left(\bigvee_{x \in X}\left(A(x) \odot \mathcal{K}^{*}\left(\top_{x}\right)(y)\right) \odot \mathcal{K}^{*}\left(\top_{y}\right)(z)\right) \\
& \left.\leq \bigvee_{x \in X}\left(A(x) \odot \mathcal{K}^{*}\left(\top_{x}\right)(z)\right)=\mathcal{K}^{*}(A)(z)\right)
\end{aligned}
$$

$(10)$

$$
\begin{aligned}
& \bigvee_{y \in X} \mathcal{K}^{*}\left(\top_{x}\right)(y) \odot \mathcal{K}^{*}\left(\top_{x}\right)(z) \leq \mathcal{K}^{*}\left(\top_{y}\right)(z) \\
& \text { iff } \bigvee_{y \in X} \mathcal{K}^{-1 *}\left(\top_{y}\right)(x) \odot \mathcal{M}\left(\top_{x}^{*}\right)(y) \leq \mathcal{K}^{*}\left(\top_{y}\right)(z) \\
& \text { iff } \mathcal{M}\left(\mathcal{K}^{-1}\left(\top_{y}\right)\right)(z) \leq \mathcal{K}^{*}\left(\top_{y}\right)(z) . \\
& \bigvee_{y \in X} \mathcal{K}^{*}\left(\top_{x}\right)(y) \odot \mathcal{K}^{*}\left(\top_{x}\right)(z) \leq \mathcal{K}^{*}\left(\top_{y}\right)(z) \\
& \text { iff } \bigvee_{y \in X} \mathcal{K}^{-1 *}\left(\top_{z}\right)(x) \odot \mathcal{M}\left(\top_{x}^{*}\right)(y) \leq \mathcal{K}^{*}\left(\top_{y}\right)(z) \\
& \text { iff } \mathcal{M}\left(\mathcal{K}^{-1}\left(\top_{z}\right)\right)(y) \leq \mathcal{K}^{-1 *}\left(\top_{z}\right)(y)
\end{aligned}
$$

Other cases and (11) are similarly proved.

Example 13. Let $\left(L=[0,1], \odot, \rightarrow,{ }^{*}\right)$ be a complete residuated lattice with the law of double negation defined by

$$
x \odot y=(x+y-1) \vee 0, x \rightarrow y=(1-x+y) \wedge 1, x^{*}=1-x .
$$

Let $X=\{x, y, z\}$ and $A, B \in L^{X}$ as follows:

$$
A(x)=0.9, A(y)=0.8, A(z)=0.3, \quad B(x)=0.3, A(y)=0.7, A(z)=0.8
$$

Define $\mathcal{K}^{*}\left(1_{x}\right)(y)=\mathcal{M}\left(1_{x}^{*}\right)(y)$ as follows

$$
\left(\begin{array}{ccc}
\mathcal{K}^{*}\left(1_{x}\right)(x)=1 & \mathcal{K}^{*}\left(1_{x}\right)(y)=0.8 & \mathcal{K}^{*}\left(1_{x}\right)(z)=0.6 \\
\mathcal{K}^{*}\left(1_{y}\right)(x)=0.7 & \mathcal{K}^{*}\left(1_{y}\right)(y)=1 & \mathcal{K}^{*}\left(1_{y}\right)(z)=0.3 \\
\mathcal{K}^{*}\left(1_{z}\right)(x)=0.5 & \mathcal{K}^{*}\left(1_{z}\right)(y)=0.6 & \mathcal{K}^{*}\left(1_{z}\right)(z)=1
\end{array}\right)
$$

(1) We have $\bigvee_{y \in X}\left(\mathcal{K}^{*}\left(1_{x}\right)(y) \odot \mathcal{K}^{*}\left(1_{y}\right)(z)=\mathcal{K}^{*}\left(1_{x}\right)(z)\right.$ and $1_{x} \leq \mathcal{K}^{*}\left(1_{x}\right)$ for all $x, y \in X$. Since $\mathcal{K}(A)(y)=\bigwedge_{x \in X}\left(A(x) \rightarrow \mathcal{K}\left(1_{x}\right)(y)\right)$ and $\mathcal{M}(A)(y)=$ $\bigvee_{x \in X}\left(\mathcal{M}\left(1_{x}^{*}\right)(y) \odot A^{*}(x)\right)$, we have

$$
\begin{aligned}
\mathcal{K}(A) & =(0.1,0.2,0.5), \mathcal{K}(B)=(0.6,0.3,0.2), \\
\mathcal{K}\left(A^{*}\right) & =(0.8,0.7,0.3), \mathcal{K}\left(B^{*}\right)=(0.3,0.5,0.7), \\
\mathcal{M}(A) & =(0.2,0.3,0.7), \mathcal{M}(B)=(0.7,0.5,0.3)
\end{aligned}
$$




$$
\mathcal{M}\left(A^{*}\right)=(0.9,0.8,0.5), \mathcal{M}\left(B^{*}\right)=(0.4,0.7,0.8) .
$$

Furthermore, by Theorem $12(3,6,9), \mathcal{K}\left(\mathcal{K}^{*}(A)\right)=\mathcal{K}(A), \mathcal{M}\left(\mathcal{M}^{*}(A)\right)=\mathcal{M}(A)$ and $\mathcal{M}(\mathcal{K}(A))=\mathcal{K}^{*}(A)$, for all $A \in X$. For $\mathcal{K}^{*}\left(1_{x}\right)=(1,0.8,0.6)$, by Theorem $12(2,6), \mathcal{K}\left(\mathcal{K}^{*}\left(1_{x}\right)=\mathcal{K}\left(1_{x}\right)=(0,0.2,0.4)\right.$. For $\mathcal{M}\left(1_{x}^{*}\right)=(1,0.8,0.6)$, by Theorem 12(2,6), $\mathcal{M}\left(\mathcal{M}^{*}\left(1_{x}^{*}\right)=\mathcal{M}\left(1_{x}^{*}\right)\right.$.

(2) We obtain $\mathcal{K}^{-1 *}\left(1_{x}\right)(y)=\mathcal{M}^{-1}\left(1_{y}^{*}\right)(x)=\mathcal{K}^{*}\left(1_{y}\right)(x)$ as follows

$$
\left(\begin{array}{ccc}
\mathcal{K}^{-1 *}\left(1_{x}\right)(x)=1 & \mathcal{K}^{-1 *}\left(1_{x}\right)(y)=0.7 & \mathcal{K}^{-1 *}\left(1_{x}\right)(z)=0.5 \\
\mathcal{K}^{-1 *}\left(1_{y}\right)(x)=0.8 & \mathcal{K}^{-1 *}\left(1_{y}\right)(y)=1 & \mathcal{K}^{-1 *}\left(1_{y}\right)(z)=0.6 \\
\mathcal{K}^{-1 *}\left(1_{z}\right)(x)=0.6 & \mathcal{K}^{-1 *}\left(1_{z}\right)(y)=0.3 & \mathcal{K}^{-1 *}\left(1_{z}\right)(z)=1
\end{array}\right)
$$

We have $\bigvee_{y \in X}\left(\mathcal{K}^{-1 *}\left(1_{x}\right)(y) \odot \mathcal{K}^{-1 *}\left(1_{y}\right)(z)=\mathcal{K}^{-1 *}\left(1_{x}\right)(z)\right.$ and $1_{x} \leq \mathcal{K}^{-1 *}\left(1_{x}\right)$ for all $x, y \in X$.

$$
\begin{aligned}
\mathcal{K}^{-1}(A) & =(0.1,0.2,0.6), \mathcal{K}^{-1}(B)=(0.5,0.3,0.2) . \\
\mathcal{K}^{-1}\left(A^{*}\right) & =(0.7,0.8,0.3), \mathcal{K}^{-1}\left(B^{*}\right)=(0.3,0.6,0.8), \\
\mathcal{M}^{-1}(A) & =(0.3,0.2,0.7), \mathcal{M}^{-1}(B)=(0.7,0.4,0.2), \\
\mathcal{M}^{-1}\left(A^{*}\right) & =(0.9,0.8,0.4), \mathcal{M}^{-1}\left(B^{*}\right)=(0.5,0.7,0.8)
\end{aligned}
$$

Furthermore, by Theorem 12(3,6,9), $\mathcal{K}^{-1}(\mathcal{K}(A))=\mathcal{K}^{*}(A), \mathcal{M}^{-1}\left(\mathcal{M}^{-1 *}(A)\right)=$ $\mathcal{M}^{-1}(A)$ and $\mathcal{M}^{-1}\left(\mathcal{K}^{-1}(A)\right)=\mathcal{K}^{-1 *}(A)$, for all $A \in X$. For $\mathcal{K}^{*}\left(1_{x}\right)=(1,0.8,0.6)$, by Theorem $12(2,6), \mathcal{K}^{-1}\left(\mathcal{K}\left(1_{x}\right)=\mathcal{K}^{*}\left(1_{x}\right)\right.$. For $\mathcal{M}^{-1}\left(1_{x}^{*}\right)=(1,0.7,0.5)$, by Theorem 12(2,6), $\mathcal{M}^{-1}\left(\mathcal{M}^{-1 *}\left(1_{x}^{*}\right)=\mathcal{M}^{-1}\left(1_{x}^{*}\right)\right.$.

(3) Since $0.6=\bigvee_{x \in X}\left(\mathcal{K}^{*}\left(1_{x}\right)(y) \odot \mathcal{K}^{*}\left(1_{x}\right)(z) \not \subset \mathcal{K}^{*}\left(1_{y}\right)(z)=0.3\right.$, then

$$
\begin{gathered}
(0.3,0,0.7)=\mathcal{K}\left(1_{y}\right) \not \leq \mathcal{K}\left(\mathcal{K}^{-1 *}\left(1_{y}\right)\right)=(0.2,0,0.4) \\
(0.8,1,0.6)=\mathcal{K}^{-1 *}\left(1_{y}\right) \not \mathcal{K}^{-1}\left(\mathcal{K}\left(1_{y}\right)\right)=(0.7,1,0.3) \\
(0.7,1,0.3)=\mathcal{K}^{*}\left(1_{y}\right) \nsupseteq \mathcal{M}\left(\mathcal{K}^{-1}\left(1_{y}\right)\right)=(0.8,1,0.6) \\
(0.7,1,0.3)=\mathcal{M}\left(1_{y}^{*}\right) \nsupseteq \mathcal{M}\left(\mathcal{M}^{-1}\left(1_{y}^{*}\right)\right)=(0.8,1,0.6) .
\end{gathered}
$$

(4) Since $0.6=\bigvee_{x \in X}\left(\mathcal{K}\left(1_{y}\right)(x) \odot \mathcal{H}\left(1_{z}\right)(x) \not \subset \mathcal{K}\left(1_{y}\right)(z)=0.3\right.$, then

$$
\begin{gathered}
(0.3,0,0.7)=\mathcal{K}\left(1_{y}\right) \not \leq \mathcal{K}^{-}\left(\mathcal{K}^{*}\left(1_{y}\right)\right)=(0.2,0,0.4) \\
(0.7,1,0.3)=\mathcal{K}^{*}\left(1_{y}\right) \not \leq \mathcal{K}\left(\mathcal{K}\left(1_{y}\right)\right)=(0.7,0.7,0.3) \\
(0.7,1,0.3)=\mathcal{K}^{*}\left(1_{y}\right) \nsupseteq \mathcal{M}^{-1}\left(\mathcal{K}\left(1_{y}\right)\right)=(0.8,1,0.6) \\
(0.7,1,0.3)=\mathcal{M}\left(1_{y}^{*}\right) \nsupseteq \mathcal{M}^{-1}\left(\mathcal{M}^{*}\left(1_{y}^{*}\right)\right)=(0.8,1,0.6) .
\end{gathered}
$$




\section{References}

[1] R. Bělohlávek,Fuzzy Relational Systems, Kluwer Academic Publishers, New York , (2002).

[2] P. Hájek, Metamathematices of Fuzzy Logic, Kluwer Academic Publishers, Dordrecht (1998).

[3] Y.C. Kim, L-join meet approximation operators to appear International Journal of Pure and Applied Mathematics. 3(5) (2013), 1193-1210.

[4] H. Lai, D. Zhang, Fuzzy preorder and fuzzy topology, Fuzzy Sets and Systems, 157 (2006), 1865-1885, doi: 10.1016/j.fss.2006.02.013.

[5] H. Lai, D. Zhang, Concept lattices of fuzzy contexts: Formal concept analysis vs. rough set theory, Int. J. Approx. Reasoning, 50 (2009), 695-707, doi: $10.1016 /$ j.ijar.2008.12.002.

[6] Z. Pawlak, Rough sets, Int. J. Comput. Inf. Sci., 11 (1982), 341-356.

[7] Z. Pawlak, Rough probability, Bull. Pol. Acad. Sci. Math., 32(1984), 607615.

[8] A. M. Radzikowska, E.E. Kerre, A comparative study of fuzy rough sets, Fuzzy Sets and Systems, 126(2002), 137-155, doi: 10.1016/so1650114(01)00032-x.

[9] Y.H. She, G.J. Wang, An axiomatic approach of fuzzy rough sets based on residuated lattices, Computers and Mathematics with Applications, 58 (2009), 189-201, doi: 10.1016/j.camwa.2009.03.100.

[10] Q. Y. Zhang, L. Fan, Continuity in quantitive domains, Fuzzy Sets and Systems, 154 (2005), 118-131, doi: 10.1016/j.fss.2005.01.007.

[11] Q. Y. Zhang, W. X. Xie, Fuzzy complete lattices, Fuzzy Sets and Systems, 160 (2009), 2275-2291, doi: 10.1016/j.fss.2008.12.001.

[12] Zhen Ming Ma, Bao Qing Hu, Topological and lattice structures of Lfuzzy rough set determined by lower and upper sets, Information Sciences, 218(2013), 194-204, doi: 10.1016/j.ins.2012.06.029. 